This manuscript was accepted and published by the Journal of Analytical and Applied Pyrolysis.

Publication data of the final, corrected work:

Várhegyi, G.; Szabó, P.; Jakab, E.; Till, F.: Least squares criteria for the kinetic evaluation of thermoanalytical experiments. Examples from a char reactivity study. J. Anal. Appl. Pyrolysis 2001, 57, 203-222. doi: 10.1016/S0165-2370(00)00113-3

\title{
Least squares criteria for the kinetic evaluation of thermo- analytical experiments. Examples from a char reactivity study.
}

\author{
Gábor Várhegyi*, Piroska Szabó, Emma Jakab and Ferenc Till
}

Research Laboratory of Materials and Environmental Chemistry, Chemical Research Center, Hungarian Academy of Sciences

*Email: varhegyi.gabor@t-online.hu or gvarhegyi@gmail.com

\begin{abstract}
The mathematical modeling of the chemical processes is a crucial problem of the thermal analysis. Simple models with few parameters seldom can describe the real complexity of the phenomena arising during the heating of the various substances. In the case of more complex models the determination of the parameters and the validation of the model require the evaluation of carefully designed experimental series. The one-by-one evaluation of the experiments is a mathematically ill-defined problem at a larger number of unknown parameters. The non-statistical experimental errors of the thermal analysis hinder the determination of a single parameter set by the simultaneous least squares evaluation of the experiments. This paper discusses several evaluation techniques for the handling of the non-statistical errors during the least squares evaluation of experimental series. The methods are illustrated by the evaluation of oxidative thermogravimetric experiments of a lignite and a coal char.
\end{abstract}

Keywords: Kinetics, least squares evaluation, thermogravimetry, char reactivity, temperature programmed combustion 


\section{Introduction}

In the early days of thermal analysis the kinetic evaluation was based on linearization techniques, plotting various functions of the experimental data against 1/T. An alternative approach was the calculation of the kinetic parameters from a few, characteristic points of the experimental curves. These methods have usually uneven sensitivity on the uncertainty of the experimental data (due to the logarithms involved) and/or do not exploit fully the information content of the experiments. Besides, these methods are restricted to relatively simple models consisting of a single equation

$$
\mathrm{d} \alpha / \mathrm{d} t=A \exp (-E / R T) f(\alpha)
$$

where $\alpha$ is the reacted mole fraction, $A$ is preexponential factor $\left(\mathrm{s}^{-1}\right), E$ activation energy $(\mathrm{kJ} / \mathrm{mol})$, and $f$ is a continuous function. Nevertheless, the traditional evaluation methods are still widely used in the literature and discussed in details in recent reviews $[1,2]$.

Obviously the real complexity of the processes requires models more sophisticated than eqn. (1). This increases the number of the unknown parameters. The determination of the higher number of unknown parameters necessitates the simultaneous evaluation of whole series of experiments, while the complexity of the model hinders the application of the usual linearization techniques. The non-linear methods of least squares, which are generally employed in other areas of science, are more promising. We may evaluate several experiments simultaneously by minimizing the following sum:

$$
S=\sum_{j=1}^{M N_{j}} \sum_{i=1} w_{i j}\left[X_{i j}{ }^{o b s}-X_{i j}{ }^{\text {calc }}\right]^{2} / N_{j}
$$

Here $X_{i j}{ }^{o b s}$ and $X_{i j}{ }^{c a l c}$ represent the experimental data (sample mass, DTG or DSC signal) and their simulated counterparts, $\mathrm{w}_{\mathrm{ij}}$ are optional weight factors, $M$ is the number of experimental curves used in the evaluations and $N_{j}$ is the number of points on the $j$ th experimental curve. The resulting fit can be expressed for each evaluated curve as

$$
\text { fit }(\%)=100\left(\sum_{i=1}^{N_{j}}\left[X_{i j}{ }^{\text {calc }}-X_{i j}{ }^{o b s}\right]^{2} / N_{j}\right)^{0.5} / X_{j}^{\text {highest }}
$$

where $X_{j}^{\text {highest }}$ is the highest absolute value of the $j$ th experimental curve. The division by $X_{j}^{\text {highest }}$ serves for normalization. The result of this approach is a set of best fitting parameters, a measure of the fit and some sort of information about the reliability of the parameters.

The use of the non-linear method of least squares in non-isothermal kinetics dates back to the pioneering work of Broido and Weinstein [3], and has been spreading gradually since then. Still, many 
controversial results appear in the literature, raising skepticism about the whole field of kinetic evaluation of non-isothermal experiments [4].

A major part of the problem can be due to the systematic errors of the thermal analysis, which hinder the mechanistic application of the non-linear least squares algorithms for series of thermoanalytical experiments. The most important errors are connected to the problems of the temperature measurements and to the self cooling/self heating of the samples during the reaction. These phenomena were extensively studied [5-7]. Bockhorn et al. also included a modeling of the heat transfer into the evaluation of the parameters of an $n$th order kinetic model [8].

In our opinion, the heat transfer problems in a real thermoanalytical apparatus are very complex. Among others, the flow of the (relatively cool) flushing gases into a dynamically heated furnace makes hard the realistic modeling. It is difficult to combine a realistic modeling of the heat transfer phenomena with complex chemical kinetic models. An alternative way is the empirical assessment of the systematic errors and the elaboration of such evaluation techniques, which allow the estimation of the kinetic parameters in spite of the inevitable experimental errors. This will be the subject of the present communication.

A recent round-robin study [9] compared thermogravimetric curves measured on the decomposition of small (0.1-5 mg) cellulose samples by up to date equipments in eight European laboratories. For a fixed value of weight loss, the scatter in the temperature measurement was about $17^{\circ} \mathrm{C}$. Measured values of the char yield laid between 3 and 11\%. In spite of these differences, the shape of the curves was similar, and the activation energies calculated from the different experiments exhibited only a moderate scattering. The deduction of these results from a theory appears to be difficult. However, the empirical description of this type of errors is relatively simple in the kinetic evaluation. Earlier we presented techniques to deal with this problem [10-14]. The aim of the present work is the assessment and comparison of several approaches. We selected a thermogravimetric char reactivity study for this purpose, where we found earlier a model valid in temperature intervals of several hundred centigrade, over a wide range of ambient gas compositions [12]. In that work one particular evaluation strategy was used. In the present work six evaluation strategies will be compared and the number of simultaneously evaluated experiments is also increased. Though we treat here only thermogravimetric curves, related works $[10,15]$ indicate that this type of evaluation is useful in other areas of thermal analysis, too.

\section{Experimental}

A computerized Perkin-Elmer TGS-2 thermobalance was used. Low sample masses $(0.4-3 \mathrm{mg})$ were employed to avoid heat and mass transfer problems. The TG curves discussed in the paper belong to 
the thermal oxidation (temperature programmed combustion) of chars prepared at $950^{\circ} \mathrm{C}$ from a French lignite from the Gardanne region and a Polish high-volatile bituminous coal (“Arkadia”) [12]. Table 1 and Figure 1 exhibit the experimental conditions of the lignite char experiments evaluated simultaneously. The experiments were carried out at atmospheric pressure, in the flow of argon - oxygen mixtures. Experiment 3 was performed in $\mathrm{CO}_{2}-\mathrm{O}_{2}$ mixture, to involve a case when the side reaction of carbonate decomposition is completely suppressed. (Note that the presence of $\mathrm{CO}_{2}$ does not affect the rate of char oxidation itself [16], and $\mathrm{Ar}$ and $\mathrm{CO}_{2}$ have similar heat conductivities.) Similar conditions were employed for the coal char experiments, except that higher isothermal temperatures were needed due to the lower reactivity of this sample. $\left(515\right.$ and $525^{\circ} \mathrm{C}$ were used in experiments 5 and 6 , respectively, while the isothermal sections of the cyclic $T(t)$ were at 495 and $510^{\circ} \mathrm{C}$.)

Table 1. Experimental conditions of seven TG experiments evaluated simultaneously in the study of Gardanne lignite char.
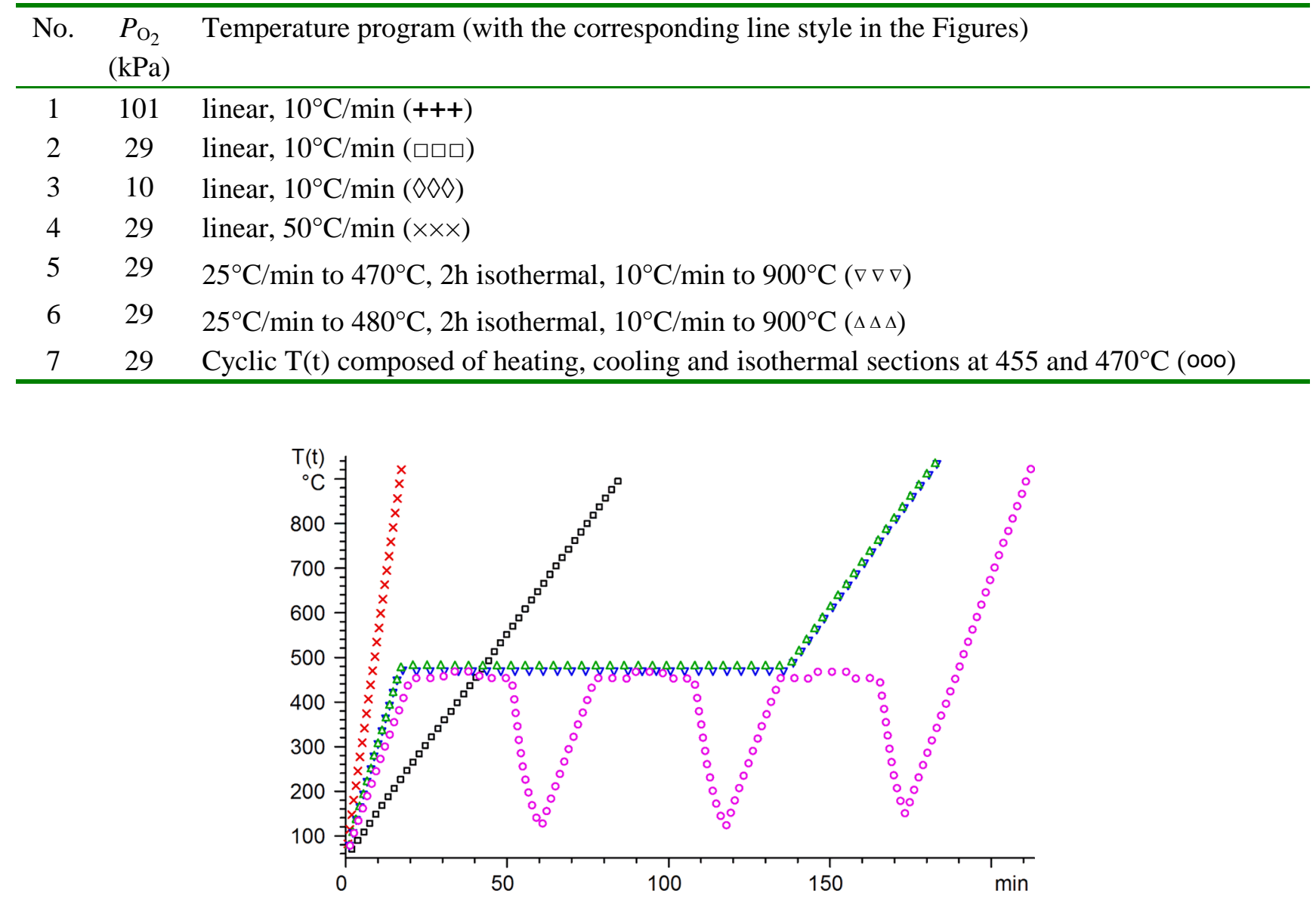

Figure 1: Temperature programs of TG experiments evaluated simultaneously in the study of Gardanne lignite char. (See Table 1.)

The experimental data were processed by FORTAN 90 and $\mathrm{C}++$ programs developed by the authors. More emphasis was placed on the safety of the iterations than on the computational speed. The differential equations of the models were solved along the experimental $\mathrm{T}(\mathrm{t})$ functions by an adaptive stepwise Runge Kutta method [17]. The least squares parameters were determined by a direct search method [17], which works reasonably on irregular surfaces, too. The results of the iterations were 
checked by restarting searches from different initial parameters. Some of the evaluations proved to be illconditioned, requesting parameter transformations [11] and the solution of the differential equations with a very high relative precision $\left(10^{-10}\right)$.

\section{Results and Discussion}

\subsection{A simple numerical example}

The systematic error of the thermoanalytical experiments depends on the experimental conditions. In this section a numerical example will be constructed. The extent of the simulated "experimental" errors reflects our experience in this field [9, 11-14].

Let us suppose that we measure a $\mathrm{TG}$ curve at a heating rate of $5^{\circ} \mathrm{C} / \mathrm{min}$ and the result can be described by first order kinetics with $E=200 \mathrm{~kJ} / \mathrm{mol}$ and $A=10^{16} \mathrm{~s}^{-1}$ :

$$
\mathrm{d} \alpha / \mathrm{d} t=A \exp (-E / R T)(1-\alpha)
$$

The numerical solution of eqn. (4) at two different heating rates, $5(\bullet \bullet \bullet)$ and $50^{\circ} \mathrm{C} / \mathrm{min}(---)$ is shown in Figure 2. In higher heating rate experiments, however, the heat and mass transfer problems are more severe than at $5^{\circ} \mathrm{C} / \mathrm{min}$, altering the shape of the experimental curves and resulting in increased thermal lag. As an example, we assumed that the sharpness of the DTG curve decreases by ca. $10 \%$ and the thermal lag increases by ca. $5^{\circ} \mathrm{C}$ at $50^{\circ} \mathrm{C} / \mathrm{min}$. We simulated a distorted "experiment" of this type by solving eqn. (4) at suitably chosen parameter values, $E=180$ and $\log A=13.95$. (See line $\times \times \times$ in Figure 2.)

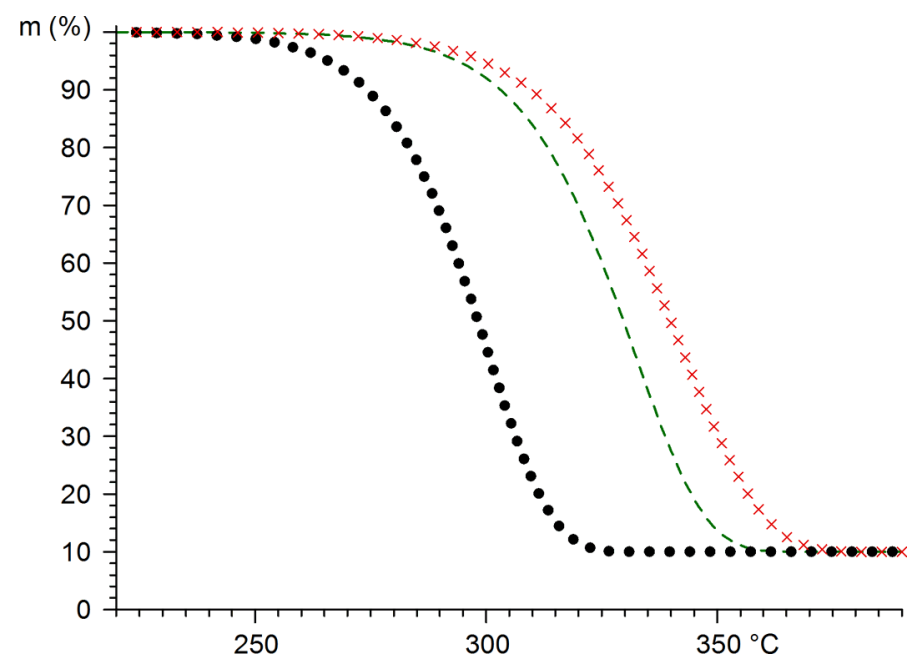

Figure 2: Construction of simulated experiments to demonstrate the concepts of the paper on simple examples. Notation: Solution of a first order kinetic differential equation at $5^{\circ} \mathrm{C} / \mathrm{min}(\bullet \bullet \bullet)$ and $50^{\circ} \mathrm{C} / \mathrm{min}(---)$; Simulated experiment at $50^{\circ} \mathrm{C} / \mathrm{min}$ assuming distortion and thermal lag $(\times \times \times)$. 
Table 2. Evaluation of simulated TG experiments by different evaluation strategies. Hypothetic systematic errors were assumed at $50^{\circ} \mathrm{C} / \mathrm{min}$ heating rate $\left(10 \%\right.$ distortion and $5^{\circ} \mathrm{C}$ thermal lag, as described in the text).

\begin{tabular}{|c|c|c|c|c|c|c|}
\hline \multirow[t]{2}{*}{ No. } & & \multirow{2}{*}{$\begin{array}{c}F i t_{\mathrm{TG}^{\mathrm{a}}} \\
\left(\% \text { of } \mathrm{m}_{0}\right)\end{array}$} & \multicolumn{2}{|c|}{$5^{\circ} \mathrm{C} / \mathrm{min}$} & \multicolumn{2}{|c|}{$50^{\circ} \mathrm{C} / \mathrm{min}$} \\
\hline & & & $\begin{array}{c}E \\
(\mathrm{~kJ} / \mathrm{mol})\end{array}$ & $\begin{array}{c}\log A \\
\left(\log \mathrm{s}^{-1}\right)\end{array}$ & $\begin{array}{c}E \\
(\mathrm{~kJ} / \mathrm{mol})\end{array}$ & $\begin{array}{c}\log A \\
\left(\log s^{-1}\right)\end{array}$ \\
\hline- & $\begin{array}{l}\text { Original parameters used } \\
\text { to simulate "observed } \\
\text { curves" }\end{array}$ & - & 200 & 16.0 & 180 & 14.0 \\
\hline $\mathrm{i}$ & $\begin{array}{l}\text { Independent evaluation } \\
\text { of the experiments }\end{array}$ & 0.0 & 200 & 16.0 & 180 & 14.0 \\
\hline ii & $\begin{array}{l}\text { Simultaneous evaluation } \\
\text { of the experiments }\end{array}$ & 1.9 & 161 & 12.4 & 161 & 12.4 \\
\hline iii & $\begin{array}{l}\text { Simultaneous evaluation } \\
\text { with varying } \log \mathrm{A}\end{array}$ & 0.5 & 188 & 14.9 & 188 & 14.7 \\
\hline iv & $\begin{array}{l}\text { Simultaneous evaluation } \\
\text { with varying } E\end{array}$ & 0.5 & 187 & 14.8 & 190 & 14.8 \\
\hline
\end{tabular}

a The deviation between the "observed" TG curves and their best fitting approximation (See equation (3)).

Let us examine what happens if we evaluate this data set (curves $\bullet \bullet \bullet$ and $\times \times \times$ ) by the method of least squares. The results are presented in Table 2. We employed here four different evaluation strategies:

i. The parameters of a first order kinetic equation can easily be determined from a single thermoanalytical curve. Accordingly, we got back the parameters used in the simulation when the experiments were evaluated independently from each other.

ii. The simultaneous evaluation of the two curves gave strongly distorted parameters (See row 1 in Table 2). In case of random, independent experimental errors this approach would be very advantageous, as described by the theory of maximum likelihood. In the present case, however, wider, flatter curves were obtained with low activation energy ( $161 \mathrm{~kJ} / \mathrm{mol})$.

iii. A shift of $\log A$ can formally describe a shift in the peak temperature [18]. Accordingly, we evaluated the two curves simultaneously, but allowed $\log A$ to have separate values for the two "experiments". In this way a good curve fitting was achieved, as shown in Figure 3a. The theoretical curves can now mimic well the characteristics of the observations. The differential curves, calculated from the first order model at the best fitting parameters, approximate reasonably the DTG curves, as shown in Figure 3b. (Here the fit of the DTG curves is demonstrated by the differences of the theoretical and experimental -dm/dT curves, since the magnitude of the - $\mathrm{dm} / \mathrm{dt}$ curves strongly depends on the heating rate.) The numerical values in Table 2 indicate that $\mathrm{E}$ and the two $\log A$ values were near to the means of the kinetic parameters used to simulate the "observations" of the example treated in this section. 

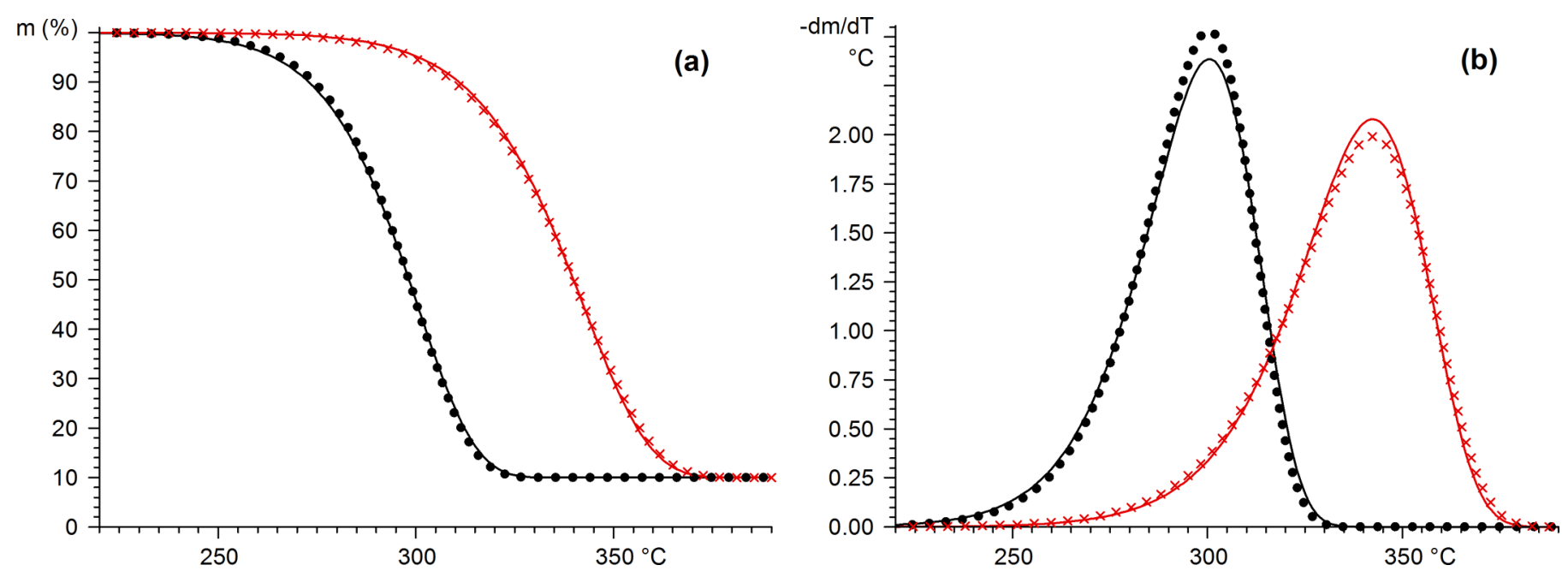

Figure 3: Evaluation of the simulated experiments of Figure 2 by strategy iii of Table 2 . The fit between the calculated curves ( - and - ) and the simulated "experiments" ( $\bullet \bullet$ and $\times \times x)$ are shown in the case of TG (a) and DTG (b) curves.

iv. A decrease in $E$ can formally describe the widening of a thermoanalytical curve and also affects the peak temperature of the simulated curves [18]. The simultaneous evaluation of the experiments with different $E$ values resulted roughly in the parameters of the previous case, indicating that small changes in the $E$ values can also approximate formally the systematic experimental errors.

Approach i proved to be the best in this simple numerical example. Approach ii, which had been optimal if the theory of maximum likelihood were applicable, gave only a rather poor performance. Approaches iii and iv resulted in a reasonable approximation of the "true" parameters. In the following section special emphasis will be given on these approximate approaches, since they proved to be useful at more complex kinetic models, too.

\subsection{A more complex example: reaction of a lignite char with oxygen}

In an earlier work [12] we studied the oxidation (controlled temperature combustion) of a lignite char. The evaluation of $30 \mathrm{TG}$ experiments measured at various experimental conditions led to the model outlined as follows.

The reacted fraction, $\alpha$, proved to be proportional to the partial pressure of oxygen, $P_{\mathrm{O}_{2}}$ :

$$
\mathrm{d} \alpha / \mathrm{d} t=A \exp (-E / R T) P_{\mathrm{O}_{2}} \mathrm{f}(\alpha)
$$

Function $f(\alpha)$ describes the change of the reactivity and surface area of the sample during the reaction. Due to the complex physical and chemical structure of the coals and lignites, there is little hope to deduce the form of $f(\alpha)$ from a reliable theory. Hence a formal approximation was introduced:

$$
\mathrm{f}(\alpha) \cong(\alpha+z)^{a}(1-\alpha)^{b}
$$


Here parameters $a, b$ and $z$ define the shape of the $\mathrm{f}(\alpha)$ function. Though the values of $a, b$ and $z$ do not have direct physical meaning, the features of the resulting $f(\alpha)$ function helps to understand better the processes going on [12].

The sample mass is obviously a linear function of the reacted fraction:

$$
m(t)=c(1-\alpha)+m_{\infty}
$$

If $m(t)$ is normalized by the initial sample mass, then $m(0)=1$ and $c=1-m_{\infty}$, where $m_{\infty}$ is the normalized sample mass at $t=\infty$. Note that $m_{\infty}$ is not equal to the ASTM ash content of the sample. It is well known that the amount of residue after the burn-off of the fuels depend on the operation conditions. (That is why special burn-off conditions are defined for the determination of the standard ash content.) In the case of the lignite char of this study, the high calcium carbonate content of the samples particularly affected the mass of the residue. Besides, a recent round-robin study [9] revealed that that the determination of the residue mass itself has substantial experimental errors in thermogravimetry. Keeping these factors in mind, we regarded $\mathrm{m}_{\infty}$ as a parameter to be determined during the least squares evaluation.

In this way we had to determine six unknown parameters: $E, \log A, a, b, z$ and $m_{\infty}$. We determined the unknown parameters by the simultaneous evaluation of seven experiments. The experiments evaluated simultaneously were selected so that their combined range of experimental conditions will be as large as possible. As Table 1 shows, slow experiments of several hours, fast experiments of a few minutes, low and high oxygen concentrations and temperature programs containing isothermal, heating and cooling sections were involved. The aim of this planning was to provide a high amount of information for the determination of the parameters.

We employed evaluation strategies similar to the ones outlined in Section 3.1. The results are summarized in Table 3. We included additional quantities to give a better overview about the performance of the various approaches. Fit $t_{\mathrm{DTG}}$ is the deviation between the theoretical $-\mathrm{dm} / \mathrm{dt}$ functions and the DTG curves. It is calculated by eqn. (3). Fit $t_{\text {DTG }}$ reflects the differences in shape of the theoretical and experimental curves much better than Fit Th. The comparison of the parameters belonging to different experimental conditions was helped by simulating curves at a standardized set of experimental conditions. Table 3 includes std. $T_{1 / 2}$, which is the temperature belonging to $\alpha=0.5$ of a curve calculated from the model at $\mathrm{dT} / \mathrm{dt}=10^{\circ} \mathrm{C} / \mathrm{min}$ and $P_{\mathrm{O}_{2}}=21 \mathrm{kPa}$ (air). It is a function of $A$ and $E$ at a given set of the $f(\alpha)$ parameters. 
Table 3. Thermal oxidation of a lignite char. Simultaneous least squares evaluation of seven TG experiments by different evaluation strategies. (The kinetic model is identical in all the six cases.)

\begin{tabular}{|c|c|c|c|c|c|c|c|c|c|c|}
\hline No. & $\begin{array}{c}\text { Scattering } \\
\text { parameters }\end{array}$ & $\begin{array}{c}\text { Fit }_{\mathrm{TG}}{ }^{\mathrm{a}} \\
\%\end{array}$ & $\begin{array}{c}\text { Fit }_{\mathrm{DTG}}{ }^{\mathrm{b}} \\
\%\end{array}$ & $\begin{array}{c}E \\
\mathrm{~kJ} / \mathrm{mol}\end{array}$ & $\begin{array}{c}\log A \\
\left(\text { ( MPa }^{-1}\right.\end{array}$ & $a$ & $b$ & $z$ & $m_{\infty}$ & $\begin{array}{c}\text { std. } T_{1 / 2}{ }^{\mathrm{c}} \\
{ }^{\circ} \mathrm{C}\end{array}$ \\
\hline$I$ & None $e^{d}$ & $1.6 \pm 0.6$ & $7.3 \pm 3.3$ & 138 & 8.1 & 1.1 & 0.2 & $10^{-5}$ & 0.59 & 553 \\
\hline II & $m_{\infty}$ & $0.8 \pm 0.3$ & $5.7 \pm 1.6$ & 155 & 9.3 & 1.4 & 0.4 & 0.06 & $0.40 \pm 0.04$ & 550 \\
\hline III & $m_{\infty}, \log A$ & $0.5 \pm 0.2$ & $4.8 \pm 1.4$ & 148 & $9.0 \pm 0.04$ & 1.6 & 0.6 & 0.06 & $0.39 \pm 0.04$ & $551 \pm 3$ \\
\hline IV & $m_{\infty}, E$ & $0.5 \pm 0.2$ & $5.0 \pm 1.5$ & $150 \pm 1$ & 9.2 & 1.7 & 0.8 & 0.13 & $0.39 \pm 0.04$ & $551 \pm 3$ \\
\hline $\mathrm{V}$ & $m_{\infty}, E, \log A$ & $0.4 \pm 0.1$ & $3.7 \pm 1.6$ & $142 \pm 14$ & $8.5 \pm 1.0$ & 1.5 & 0.4 & 0.03 & $0.40 \pm 0.04$ & $551 \pm 7$ \\
\hline$V I$ & $A l l^{d}$ & $0.1 \pm 0.1$ & $1.6 \pm 0.7$ & $126 \pm 21$ & $7.5 \pm 1.4$ & $1.5 \pm 0.6$ & $0.5 \pm 0.3$ & $0.02 \pm 0.02$ & $0.40 \pm 0.01$ & $568 \pm 34$ \\
\hline
\end{tabular}

a $\%$ of initial sample mass.

b $\%$ of the highest value on the given DTG curve.

c The temperature belonging to $\alpha=0.5$ of a curve calculated from the model at $21 \% \mathrm{O}_{2}$ and $\mathrm{dT} / \mathrm{dt}=10^{\circ} \mathrm{C} / \mathrm{min}$.

${ }^{d}$ Italic typography denotes rejected results. 
The approaches applied are arranged here in the order of the number of unknown parameters, as follows.

I. The same $E, \log A, a, b, z$ and $m_{\infty}$ parameters were searched for all experiments. In this case the fit was poor, due to the non-statistical nature of the experimental errors. The theoretical curves did not mimic well the shape of the experimental curves. Particularly, the theoretical derivative curves were wider than the observed DTG peaks, in the same way as in the corresponding evaluation of Section 3.1, above.

II. In the second case each experiments had its own $m_{\infty}$ parameter. The seven experiments were evaluated simultaneously. 12 unknown parameters were determined: $E, \log A, a, b, z$ and seven different values for $m_{\infty}$. This approach helped describe the variation of the ash yield, resulting in a better fit and a closer match between the shape of the calculated and experimental curves.
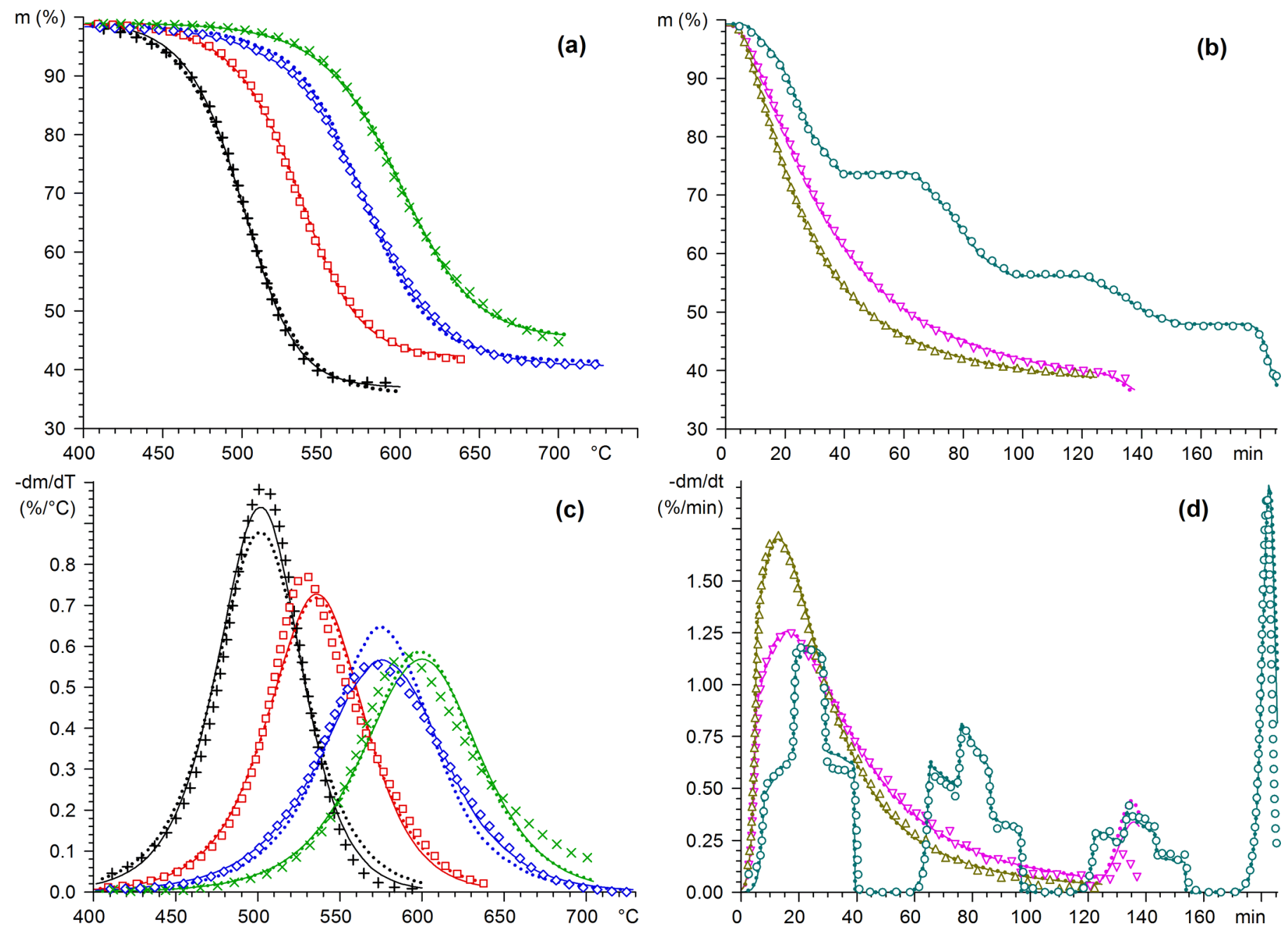

Figure 4: Simultaneous evaluation of seven lignite char oxidation experiments. The constant heating rate experiments $(+, \square, \diamond, \times)$ and the ones obtained at non-linear $\mathrm{T}(\mathrm{t})$ programs $(\nabla, \Delta$, and $\circ)$ are shown in different panels for a better visibility. The figure demonstrates how the results of strategies III ( $\cdots$ ) and V ( -$)$ fit the experimental TG $(a, b)$ and DTG (c, d) curves. 
III. When we also allowed $\log A$ to vary between the experiments, the fit improved further, since the theoretical curves were able to mimic the constant components of the temperature errors. Lines...$\cdot$ in Figure 4 represent the $m(t)$ curves and their derivative functions calculated from these results. Panel (c) illustrate that the variation of $\log A$ cannot mimic the variation of the peak distortion with the experimental conditions. The DTG peak top in pure oxygen $(+++)$, for example, is much sharper than the curve calculated from this approach (...), indicating a considerable overheating of the sample in this section. The $E$ value determined in this approach had to approximate simultaneously the sharpness of all the seven experiments, resulting in too sharp derivative curves in other cases.

IV. When we allowed $E$ and $m_{\infty}$ to vary between the experiments, the resulting parameters and the fit was similar to the previous case, indicating that changes in $\log A$ and in $E$ can describe the systematic errors roughly to the same extent. It may be worth emphasizing that we did not assume a change in a chemical mechanism as a function of the experimental conditions. The scattering of the $E$ values in this type of calculations serves only to describe formally the experimental errors.

V. The variation of $\log A, E$ and $m_{\infty}$ between the experiments further improved the fit, due to the 24 parameters optimized in this case. ( $a, b$ and $z$ were common for the whole data set, while separate $\log A$, $E$ and $m_{\infty}$ values were searched for each experiment.) Still the results were coherent. The scattering of E was $10 \%$, which is close to the result of a recent round-robin study on a much simpler system [9]. The deviation of the $s t d$. $T_{1 / 2}$ values is closely connected to the deviations of $\log A$ and $E$. Its relatively high value, $7^{\circ} \mathrm{C}$, can be due to the combination of several factors: (i) the high reaction heat of char combustion alters the true sample temperature from its measured values; (ii) we employed extremely varying experimental conditions; (iii) the model is approximate. The $m(t)$ curves calculated from these results and their derivative functions are represented by solid lines in Figure 4.

VI. When the experiments were evaluated independently from each other, the scattering of the parameters increased to very high levels. The high number of optimized parameters allowed a very close approximation of the observations, without emphasizing the common features in the series of experiments. It is well demonstrated by the high deviation $\left(34^{\circ} \mathrm{C}\right)$ of the $s t d . T_{1 / 2}$ values.

It is well worth observing that approaches II - V gave results close to each other. The mean $E$ values were 155, 148, 150 and $142 \mathrm{~kJ} / \mathrm{mol}$, respectively, differing from each other by 1 - 9\%. These differences are within the reliability limits of the kinetic evaluation of the thermogravimetric curves [9]. The $f(\alpha)$ functions resulting form these evaluations are also similar, as shown in Figure 5. Unfortunately, no theory can tell which is the "best" among these approaches. 


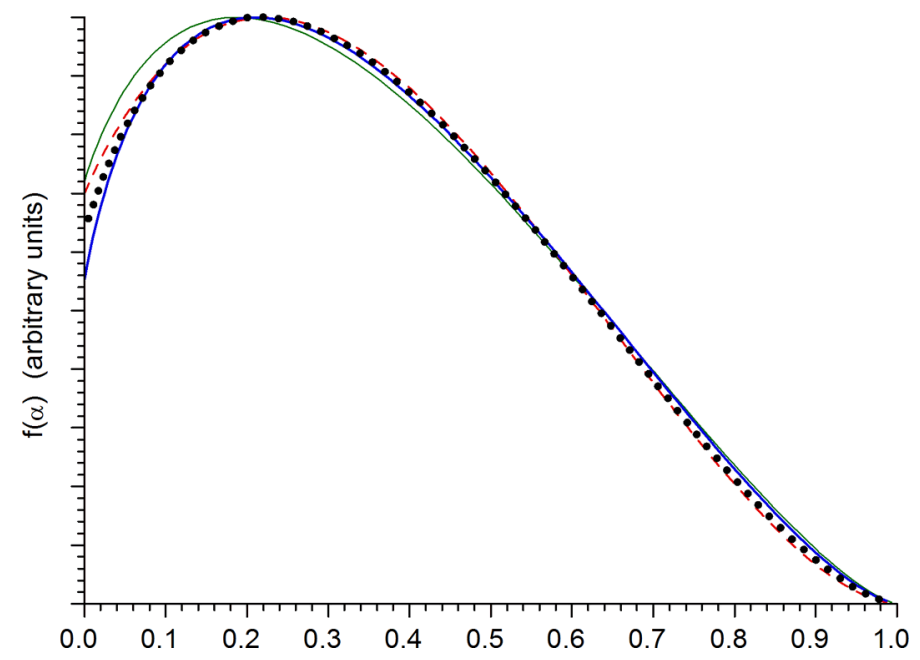

Figure 5: $\mathrm{f}(\alpha)$ functions obtained for the lignite char by strategies II (-), III (•••), IV (- - -) and V (-).

\subsection{Example for a larger number of unknown parameters: reaction of a coal char with oxygen}

Chars are usually heterogeneous substances. The model outlined in the previous paragraph can formally approximate the physical distribution of particle size, porosity, etc., due to the flexibility of the $f(\alpha)$ function defined by eqn. (6). In case of coal chars, however, more pronounced inhomogeneities are also observed which manifest in two partly overlapping peaks on the DTG curve [19]. Silveston [20] explained this phenomenon by postulating two carbon components of different reactivity. Accordingly, we describe this case by assuming two carbon components with reacted fractions $\alpha_{1}$ and $\alpha_{2}$ :

$$
\begin{aligned}
& \mathrm{d} \alpha_{1} / \mathrm{d} t=A_{1} \exp \left(-E_{1} / R T\right) P_{\mathrm{O}_{2}} \mathrm{f}_{1}\left(\alpha_{1}\right) \\
& \mathrm{d} \alpha_{2} / \mathrm{d} t=A_{2} \exp \left(-E_{2} / R T\right) P_{\mathrm{O}_{2}} \mathrm{f}_{2}\left(\alpha_{2}\right) \\
& \mathrm{f}_{1}\left(\alpha_{1}\right) \cong\left(\alpha_{1}+z_{1}\right)^{a_{1}}\left(1-\alpha_{1}\right)^{b_{1}} \\
& \mathrm{f}_{2}\left(\alpha_{2}\right) \cong\left(\alpha_{2}+z_{2}\right)^{a_{2}}\left(1-\alpha_{2}\right)^{b_{2}}
\end{aligned}
$$

The sample mass is the linear combination of $\alpha_{1}(t)$ and $\alpha_{2}(t)$ :

$$
m(t)=c_{1}\left(1-\alpha_{1}\right)+c_{2}\left(1-\alpha_{2}\right)+m_{\infty}
$$

where $c_{1}$ and $c_{2}$ are the mass loss of component 1 and 2 , respectively, during the reactions. The substitution of the initial conditions, $m(0)=1$ and $\alpha_{1}(0)=\alpha_{2}(0)$ into eqn. (12) yields

$$
c_{1}+c_{2}+m_{\infty}=1
$$

In the evaluations by this model we used the $c_{1} / c_{2}$ ratio and $m_{\infty}$ as independent parameters. (They define $c_{1}, c_{2}$ and $m_{\infty}$ through eqn. (13).) The actual values of $c_{1} / c_{2}$ and $m_{\infty}$ are influenced by the fact that the ash yield of the carbons depends on the procedural parameters of the burn off. Parameter $m_{\infty}$ is also affected by the usual experimental errors of thermogravimetry, as discussed above. 
Table 4. Thermal oxidation of a coal char exhibiting two partly overlapping steps on the thermogravimetric curves. Simultaneous least squares evaluation of seven TG experiments by different evaluation strategies. (The kinetic model is identical in all the seven cases. See Table 3 for the notation employed.)

\begin{tabular}{|c|c|c|c|c|c|c|c|c|c|c|c|}
\hline No. & $\begin{array}{l}\text { Scattering } \\
\text { parameters }\end{array}$ & $\begin{array}{c}\text { Fit }_{\mathrm{TG}} \\
\%\end{array}$ & $\begin{array}{c}\text { Fit }_{\mathrm{DTG}} \\
\%\end{array}$ & $\begin{array}{c}E \\
\mathrm{~kJ} / \mathrm{mol}\end{array}$ & $\begin{array}{c}\log A \\
\left(\mathrm{~s} \mathrm{MPa}^{-1}\right.\end{array}$ & $a$ & $b$ & $z$ & $c_{1} / c_{2}$ & $\begin{array}{l}m_{\infty} \\
{ }^{\circ} \mathrm{C}\end{array}$ & $\begin{array}{l}\text { std. } T_{1 / 2} \\
{ }^{\circ} \mathrm{C}\end{array}$ \\
\hline \multirow[t]{2}{*}{$I$} & \multirow[t]{2}{*}{ None } & \multirow[t]{2}{*}{$2.3 \pm 0.6$} & \multirow[t]{2}{*}{$7.1 \pm 2.3$} & 129 & 7.4 & 1.6 & 0.5 & $10^{-5}$ & \multirow[t]{2}{*}{4.43} & \multirow[t]{2}{*}{0.11} & 592 \\
\hline & & & & 70 & 3.0 & 1.2 & 0.5 & 0.06 & & & 672 \\
\hline \multirow[t]{2}{*}{ II } & \multirow[t]{2}{*}{$m_{\infty}$} & \multirow[t]{2}{*}{$1.7 \pm 0.5$} & \multirow[t]{2}{*}{$5.0 \pm 1.2$} & 140 & 8.4 & 1.6 & 0.7 & 0.001 & \multirow[t]{2}{*}{0.86} & \multirow[t]{2}{*}{$0.15 \pm 0.03$} & 579 \\
\hline & & & & 110 & 5.6 & 1.0 & 0.3 & $10^{-5}$ & & & 621 \\
\hline \multirow[t]{2}{*}{ III } & \multirow{2}{*}{ 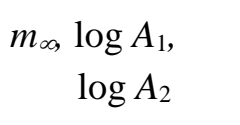 } & \multirow[t]{2}{*}{$0.3 \pm 0.1$} & \multirow[t]{2}{*}{$2.4 \pm 1.0$} & 131 & $7.9 \pm 0.1$ & 1.7 & 0.7 & 0.02 & \multirow[t]{2}{*}{1.23} & \multirow[t]{2}{*}{$0.15 \pm 0.04$} & $580 \pm 6$ \\
\hline & & & & 110 & $5.8 \pm 0.1$ & 1.1 & 0.5 & 0.07 & & & $625 \pm 10$ \\
\hline \multirow[t]{2}{*}{ IV } & \multirow{2}{*}{$\begin{array}{r}m_{\infty,} E_{1} \\
E_{2}\end{array}$} & \multirow[t]{2}{*}{$0.3 \pm 0.1$} & \multirow[t]{2}{*}{$2.3 \pm 1.0$} & $133 \pm 1$ & 8.0 & 1.6 & 0.8 & 0.01 & \multirow[t]{2}{*}{1.18} & \multirow[t]{2}{*}{$0.15 \pm 0.03$} & $578 \pm 6$ \\
\hline & & & & $108 \pm 1$ & 5.7 & 1.2 & 0.5 & 0.002 & & & $627 \pm 9$ \\
\hline \multirow[t]{2}{*}{$\mathrm{V}$} & \multirow{2}{*}{$\begin{array}{r}m_{\infty}, \log A_{1}, E_{1} \\
\log A_{2,} E_{2}\end{array}$} & \multirow[t]{2}{*}{$0.1 \pm 0.1$} & \multirow[t]{2}{*}{$1.0 \pm 0.5$} & $151 \pm 22$ & $9.1 \pm 1.5$ & 1.5 & 0.7 & 0.001 & \multirow[t]{2}{*}{0.90} & \multirow[t]{2}{*}{$0.15 \pm 0.03$} & $574 \pm 7$ \\
\hline & & & & $108 \pm 22$ & $5.6 \pm 1.5$ & 1.0 & 0.4 & $10^{-4}$ & & & $621 \pm 16$ \\
\hline \multirow[t]{2}{*}{$V I$} & \multirow{2}{*}{$\begin{array}{l}\text { All } \\
\text { (Iteration \#1) }\end{array}$} & \multirow[t]{2}{*}{$0.1 \pm 0.0$} & \multirow[t]{2}{*}{$0.8 \pm 0.4$} & $139 \pm 24$ & $8.4 \pm 1.4$ & $1.6 \pm 0.3$ & $0.7 \pm 0.1$ & $10^{-4} \pm 10^{-4}$ & \multirow[t]{2}{*}{$1.07 \pm 0.44$} & \multirow[t]{2}{*}{$0.15 \pm 0.03$} & $574 \pm 15$ \\
\hline & & & & $83 \pm 43$ & $4.1 \pm 2.9$ & $1.0 \pm 0.1$ & $0.5 \pm 0.1$ & $0.02 \pm 0.04$ & & & $662 \pm 68$ \\
\hline$V I I$ & All & $0.1 \pm 0.1$ & $0.8 \pm 0.5$ & $143 \pm 43$ & $8.6 \pm 2.9$ & $1.7 \pm 0.2$ & $0.7 \pm 0.1$ & $0.001 \pm 0.002$ & $1.06 \pm 0.28$ & $0.15 \pm 0.03$ & $589 \pm 15$ \\
\hline & (Iteration \#2) & & & $118 \pm 23$ & $6.2 \pm 1.3$ & $1.0 \pm 0.1$ & $0.4 \pm 0.2$ & $0.06 \pm 0.14$ & & & $629 \pm 20$ \\
\hline
\end{tabular}


The evaluation methods are presented in the order of the number of unknown parameters, as in the previous section. The results are compiled in Table 4 .

I. The same $E, \log A, a, b, z$ and $m_{\infty}$ parameters were used for all experiments. This approach resulted in a poor fit, similarly to the previous example.

II. When each experiments had its own $m_{\infty}$ parameter, the fit slightly increased and the parameters were close to the results of approaches III and IV, discussed below.

III. When we also allowed $\log A_{1}$ and $\log A_{2}$ to vary between the experiments, the fit improved further, since the theoretical curves were able to mimic the constant components of the temperature errors. Lines... in Figure 6 represent the $m(t)$ curves and their derivative functions calculated from these results. It may be worth observing in panel (c) that this approach did not mimic the sharpness at the top of the DTG peaks of experiments +++ and $\square \square \square$, indicating that the high reaction rates, accompanied by high heat release, resulted in significant overheating of the samples in these experiments.
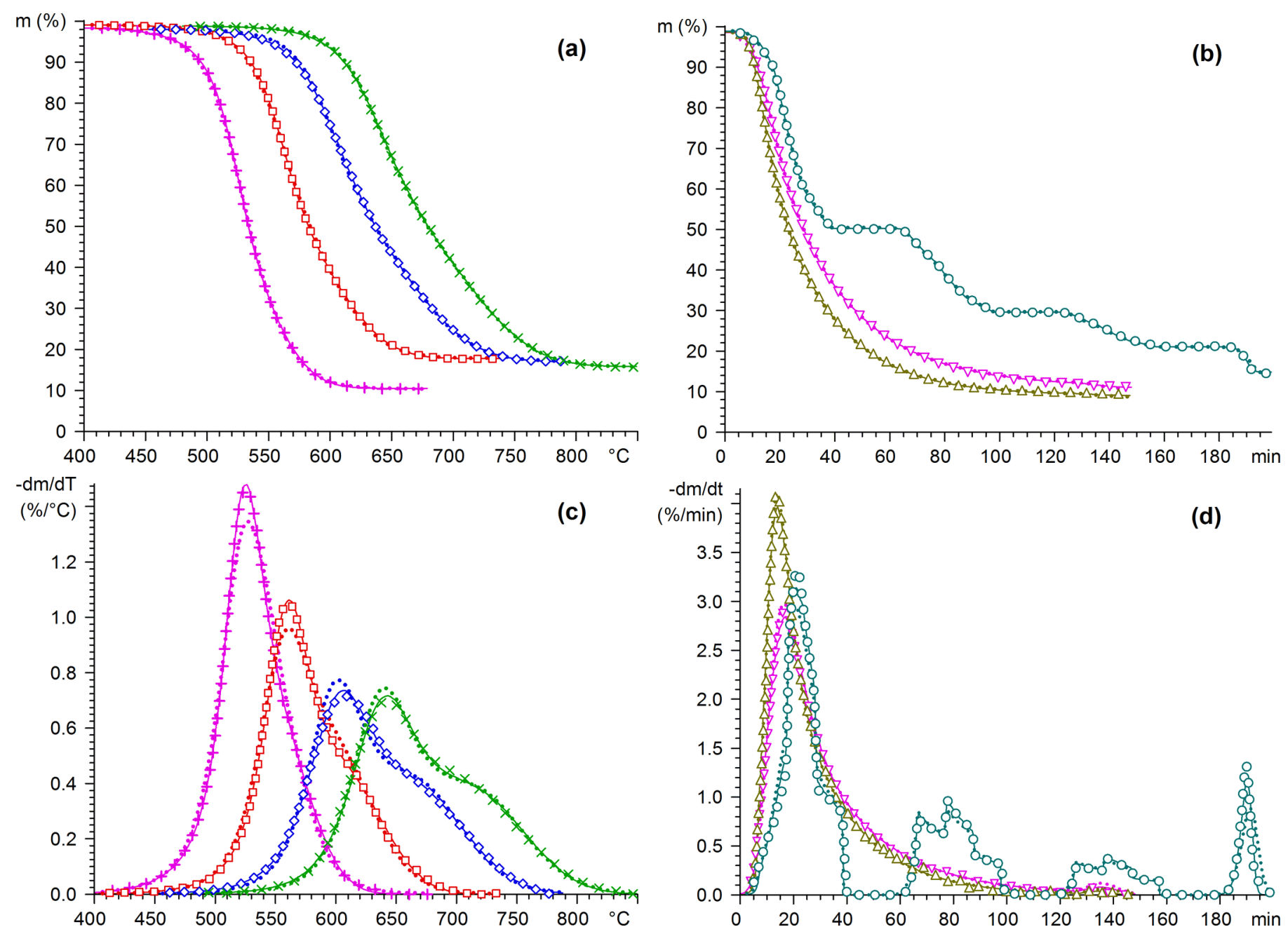

Figure 6: Simultaneous evaluation of seven coal char oxidation experiments. The constant heating rate experiments $(+, \square, \diamond, x)$ and the ones obtained at non-linear $\mathrm{T}(\mathrm{t})$ programs $(\nabla, \Delta$, and 0$)$ are shown in different panels for a better visibility. The figure demonstrates how the results of strategies III $(\cdots)$ and V $(-)$ fit the experimental TG (a, b) and DTG (c, d) curves. 
IV. When we allowed $E_{1}, E_{2}$ and $m_{\infty}$ to vary between the experiments, the resulting parameters and the fit was similar to the previous case, indicating that changes in $\log A$ and in $E$ can describe the systematic errors roughly to the same extent.

V. The variation of $\log \log A_{1}, \log A_{2}, E_{1}, E_{2}$ and $m_{\infty}$ between the experiments further improved the fit, due to the $42(!)$ parameters optimized in this case. The calculated $m(t)$ and $-\mathrm{d} m / \mathrm{d} t$ functions are represented by solid lines in Figure 6. The resulting mean parameters were similar to those of approaches II - IV, but the scattering of the parameters became much higher.

VI. When the experiments were evaluated independently from each other, the system became illdefined and the results depended on the choice of the initial parameter values of the iteration. Table 4 shows two different sets of optimal parameters, which were obtained by starting the iteration from the results of approaches I and III, respectively. The scattering of the parameters increased to very high levels, while the fit remained roughly the same as in the case of approach V.

The results of approaches I and VI were rejected. Approaches II - V resulted in similar mean parameter values. The corresponding $f(\alpha)$ functions are also similar, as shown in Figure 7. The differences between the results of approaches II - V indicate that a high number of parameters in a complex model can only approximately be determined.
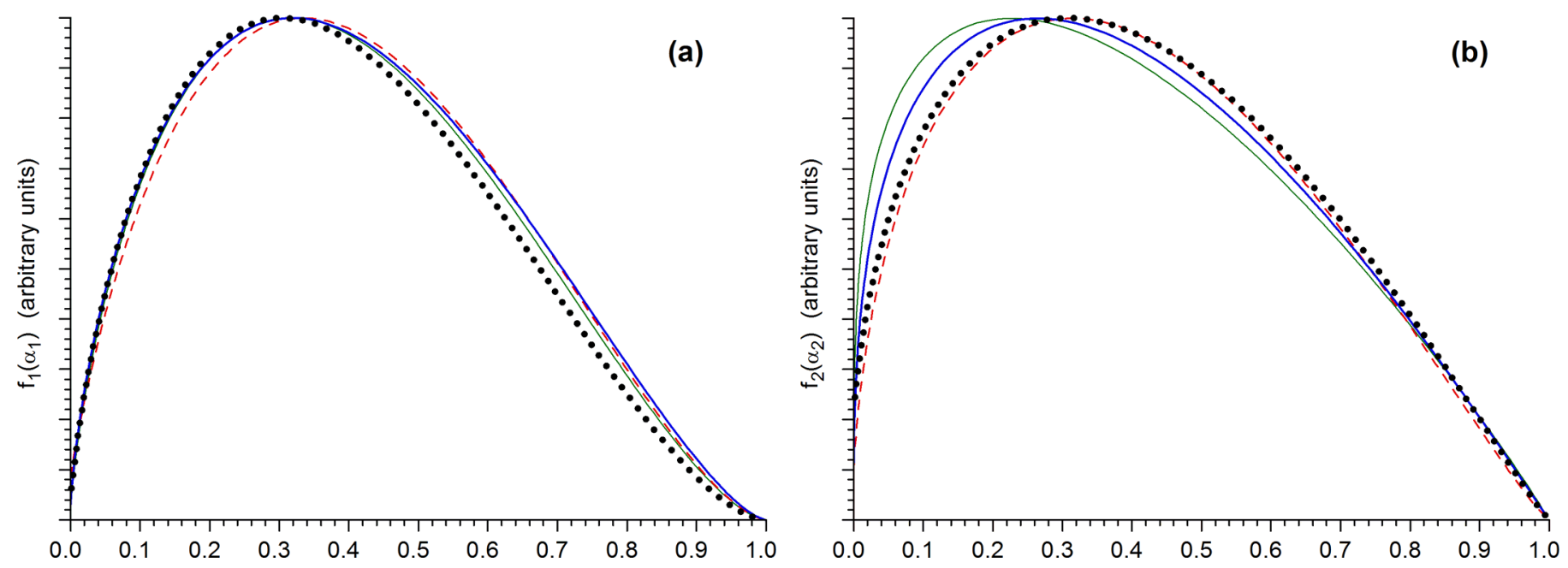

Figure 7: $f_{1}\left(\alpha_{1}\right)(a)$ and $f_{2}\left(\alpha_{2}\right)$ (b) functions obtained for the coal char by strategies II (-), III ( $\cdots$ ), IV (- - -) and $\mathrm{V}($ (一).

\subsection{Which of the above strategies can be recommended for general use?}

Approach II of paragraphs 3.2 and 3.3 resulted in poor fits. The corresponding fit $t_{\mathrm{TG}}$ in Table 4 is five times worse than the fit TG values of approaches III and IV. The poor fit may hinder the full exploitation of the information contained in the fine details of the shape of the curves. This technique handles only the variation $m_{\infty}$, which is obviously not the most important experimental error. 
On the other hand, approach $\mathrm{V}$ is based on the optimization of an unusually high number of parameters, requiring a considerable amount of experimental information. In the cases treated in the paper we were able to collect data over a sufficiently wide domain of experimental conditions. However, not all reactions permit the execution of experiments in such wide domains. Besides, future models may have more unknown parameters than the ones treated in this paper. Hence approach V cannot be recommended as a safe evaluation method for general use.

In this way the selection is narrowed to approaches III and IV, which exhibited roughly the same performance. The effects of approach III are easy to visualize: if each experiment has its own $\log A$ value(s), then the theoretical curves can follow the temperature-shift errors of the experiments (i.e. those components of the temperature errors which are approximately constant in the domain of a given DTG peak). This facilitates the interpretation of the scattering of the $\log A$ values resulting from the calculation. There is a simple mathematical equation connecting a change of $\log A$ to the magnitude of a shift of the peak temperature [12]. It is more difficult to survey the effects of approach IV, since an alteration of an $E$ value changes the width and the position of the corresponding DTG peak in different directions. Hence the easier interpretation of the results favors approach III.

\section{Conclusions}

1. The one-by-one evaluation of the thermogravimetric experiments by the method of least squares worked well with a low number of unknown parameters, but failed to give acceptable results when the kinetic model contained six or more unknowns.

2. The determination of a unique set of kinetic parameters by the simultaneous least squares evaluation of several experiments resulted in poor curve fitting and parameters strongly biased by the non-statistical experimental errors of thermal analysis.

3. The variation of the non-statistical experimental errors within a series of experiments can formally be described by allowing a selected number of kinetic parameters to have different values in the different experiment. A moderate scattering of $\log A$ and/or $E$ described well the variation of the thermal lag and peak widening caused by the different systematic temperature errors in the different experiments.

4. The uncertainty of the ash yield and the inhomogeneity of the industrial samples can also be described by allowing the corresponding parameters of the model to scatter within a series of experiments.

5. This method is a compromise between the one-by-one evaluation of the curves and the regular simultaneous least squares evaluation. From a mathematical point of view, the determination of several parameters via the one-by-one least squares evaluation of the thermogravimetric curves is an ill-defined 
problem. We can replace this ill-conditioned problem by a similar, but mathematically better-defined problem.

6. The above approaches cannot yield a "best" solution. The acceptance or rejection of the results should be based on careful pondering several factors, including: the quality of the fit; the consistency of the obtained parameters within the series of experiments; and the accordance of the results with other available information sources.

7. The quantitative characterization of the fit should not be based on a singly quantity. Beside fit $t_{T G}$, which is closely related to the objective function of the evaluation, it is well worth to calculate fit $t_{D T G}$, too, since it reflects better the similarities or differences in the peak shapes. The comparison of the parameters belonging to different experimental conditions is helped by simulating curves at the same experimental conditions (see $s t d . T_{1 / 2}$ in the paper).

8. The evaluation strategies differing in the selection of the common and scattering parameters provided similar results in the calculations of the present paper. The differences between the results were within the reliability limits of the kinetic evaluation of the thermogravimetric curves. However, other aspects (the safety of the evaluation and the interpretability of the results) favor approach III. In this method each experiment has its own $\log A$ value(s), consequently the theoretical curves can follow those components of the temperature errors which are approximately constant in the domain of a given DTG peak.

9. The authors attribute a particular importance to the question: does the given model with the given set of parameters describe the results over a sufficiently wide interval of the experimental conditions? To achieve it, series of experiments involving strongly differing $\mathrm{T}(\mathrm{t})$ programs, carrier gas compositions, etc. are suggested.

\section{Acknowledgements}

This work was supported by the Hungarian National Research Fund (OTKA T025347 and T 025341) and by the Academic Research Fund (AKP 96-93/23).

\section{REFERENCES}

[1] S. Gaur, T. B. Reed, Thermal data for natural and synthetic fuels. Marcel Dekker, 1998.

[2] A. K. Burnham, R. L. Braun, Global kinetic analysis of complex materials. Energy Fuels, 13 (1999) 1 - 22.

[3] A. Broido, M. Weinstein, Kinetics of solid-phase cellulose pyrolysis. In Proc. 3rd Internat. Conf. Thermal Anal., Wiedemann, Ed., Birkhauser Verlag, Basel, 1971, pp.285-296.

[4] M. Maciejewski, Somewhere between fiction and reality. The usefulness of kinetic data of solid-state reactions. J. Thermal. Anal., 38 (1992) 51-70.

[5] Gy. Pokol, S. Gál, E. Pungor, The effect of heat transport within the sample on the shape of the thermoanalytical curves. Thermochim. Acta, 105 (1986) 313-331. 
[6] R. Narayan, M. J. Antal, Jr., Thermal lag, fusion and compensation effect during biomass pyrolysis. Energy Fuels, 35 (1996) 1711-1721.

[7] H. Bockhorn, A. Hornung, U. Hornung, P. Jakobströer, Modelling of isothermal and dynamic pyrolysis of plastics considering non-homogeneous temperature distribution and detailed degradation mechanism. $J$. Anal. Apl. Pyrolysis, 49 (1999) 53-74.

[8] H. Bockhorn, A. Hornung, U. Hornung, Mechanism and kinetics of thermal decomposition of plastics from isothermal and dynamic measurements. J. Anal. Appl. Pyrolysis, 50 (1999) 77-101.

[9] M. Grønli, M. J. Antal, Jr., G. Várhegyi, A round-robin study of cellulose pyrolysis kinetics by thermogravimetry. Ind. Eng. Chem. Res. 38 (1999) 2238-2244.

[10] G. Várhegyi, P. Szabó, W. S. L. Mok, M. J. Antal, Jr., Kinetics of the thermal decomposition of cellulose in sealed vessels at elevated pressures. Effects of the presence of water on the reaction mechanism. J. Anal. Appl. Pyrolysis, 26 (1993) 159-174.

[11] G. Várhegyi, E. Jakab, M. J. Antal, Jr., Is the Broido - Shafizadeh model for cellulose pyrolysis true? Energy Fuels, 8 (1994) 1345-1352.

[12] G. Várhegyi, P. Szabó, E. Jakab, F. Till, J-R. Richard, Mathematical modeling of char reactivity in Ar- $\mathrm{O}_{2}$ and $\mathrm{CO}_{2}-\mathrm{O}_{2}$ mixtures. Energy Fuels, 10 (1996) 1208-1214.

[13] M. J. Antal, Jr., G. Várhegyi, E. Jakab, Cellulose Pyrolysis Kinetics: Revisited. Ind. Eng. Chem. Res., 37 (1998) 1267-1275.

[14] M. J. Antal, Jr., G. Várhegyi, Impact of systematic errors on the determination of cellulose pyrolysis kinetics. Energy Fuels, 11 (1997) 1309-1310.

[15] I. Virág, L. Pöppl., G. Várhegyi, Kinetic study of rapidly quenched $\mathrm{Ni}_{81} \mathrm{P}_{19}$ amorphous alloys. Thermochim. Acta, 351 (2000) 79-84.

[16] G. Várhegyi, F. Till, Comparison of temperature programmed char combustion in $\mathrm{CO}_{2}-\mathrm{O}_{2}$ and $\mathrm{Ar}-\mathrm{O}_{2}$ mixtures at elevated pressure. Energy Fuels, 13 (1999) 539-540.

[17] W. H. Press, B. P. Flannery, S. A. Teukolsky, W. T. Vetterling, Numerical Recipes. The Art of Scientific Computing. Cambridge University Press, Cambridge (U.K.), 1986.

[18] G. Várhegyi, T. Székely, Reaction kinetics in thermal analysis: The sensitivity of kinetic equations to experimental errors. A mathematical analysis. Thermochim. Acta, 57 (1982) 13-28.

[19] K. Miura, M. Makino, P. L. Silveston, Two-step gasification of flash pyrolysis and hydropyrolysis chars from low-rank Canadian Coals. Energy Fuels 4 (1990) 24-27.

[20] P. L. Silveston, Analysis of 2-step reaction observed in air gasification of coal through a temperatureprogrammed reaction technique - Comment. Energy Fuels 5 (1991) 933-934. 